\title{
THE CLINICAL SIGNIFICANCE AND TREATMENT OF LESIONS OF THE INTERVERTEBRAL DISK
}

\author{
BY \\ NORMAN CAPENER \\ Orthopaedic Surgeon, Princess Elizabeth Orthopaedic Hospital, Exeter, and Mount Gold Orthopaedic \\ Hospital, Plymouth, and Consultant Orthopaedic Surgeon, Royal Devon and Exeter Hospital, and \\ Ministry of Pensions Hospital, Taunton
}

The following discussion of the " disk problem ", although based upon a paper read before the Orthopaedic Section of the British Medical Association in June 1948, should be regarded as complementary to the anatomical and mechanical study of the lumbo-sacral region published previously in the Annals of the Rheumatic Diseases (Capener, 1944). My thesis is that, important as the retropulsion of an intervertebral disk may be in the causation of sciatica, nevertheless there is a large group of associated disturbances which merit a long course of conservative treatment before it is justified to carry out surgical intervention. The treatment that I am concerned to emphasize is the use of the plaster-jacket method of relative immobilization, associated with a carefully planned course of after-treatment by physical methods. I would go further, and say that we need also to consider some additional factors relating to the causation of lumbo-sacral derangements. I refer to fatigue and debility (both psychological and physical) which seem so prevalent at the present time; these factors may have an important bearing on their apparently greatly increased incidence today.

It is important that we should be clear about what we are discussing. The layman wants labels for the diseases he suffers from, and we medical men are driven to supply them. In our ignorance we apply such terms as sciatica, lumbago, rheumatism, fibrositis and, if we belong to a particular school of thought, we may even talk about osteopathic lesions. I would, however, like to ask whether the average doctor is any wiser when he sends a patient to hospital with the diagnosis of "disk lesion" or "slipped disk". If, as we have been led to believe, such disturbances mean operation, then I think that we are doing our patients a great disservice by using such labels, which detract from the essential problems with which we have to deal. I will expose the looseness of the term "lesion of the intervertebral disk" (applied.as it often is only to posterior protrusion of the disk) by the reminder that almost every pathological process affecting the spine involves, more or less, the intervertebral disks. This is particularly true of spinal tuberculosis, which is commonly mistaken, in its early stages, for such mechanical lesions of the intervertebral disks. The intervertebral disks are merely individual articulations of the spine and, as with the other joints of the body, have an equally varied pathology ranging from congenital anomalies, developmental disturbances, infective arthritis, chronic deforming arthritis, metabolic disorders, and traumatic defects, to neoplasms. The differential diagnosis of traumatic lesions of the intervertebral disk must consider all these possibilities as well as other disturbances of the vertebral skeleton, the spinal cord, the viscera, the rest of the locomotor system, and the brain; this is what makes the present subject so fascinating, yet nevertheless impossible of adequate discussion in the space here available.

\section{Anatomy and Pathology}

An important concept to grasp is that the intervertebral joints do not have the individuality of function that is possessed, say, by the hip joint or shoulder joint. Each intervertebral disk, of which there are twenty-three, is a link in the chain of the vertebral skeleton, giving both flexibility and stability to the whole. In life, movement of one vertebra upon another can only take place as part of a movement of the spine as a whole. Movement may be more marked in one region of the spine, but it can scarcely occur without movement elsewhere. The importance of the disk system in the spine is revealed when we appreciate that altogether the disks comprise one-quarter of its movable length. Pathological disturbances of the mechanics of the spine for the reasons already mentioned rarely involve one disk alone. The individual disks are thicker where mobility is greatest. These regions, the cervical and lumbar respectively, appear particularly prone to certain traumatic lesions.

It is as well that we should refresh our minds upon the nature and structure of an intervertebral disk. It must first be realized that each one is only part of an intervertebral articulation. We are dealing with a synchondrosis associated with two 
posterior apophyseal diarthroses which are complementary to it. There is, therefore, a triple joint and not unnaturally we should expect that disturbances of one section of the joint should also involve the others more or less. This is well demonstrated in the profound disturbances of spondylolisthesis, and is probably true in a less marked degree in spondylolysis, fractures of the articular processes, and in arthritic degeneration of the posterior joints. The intervertebral synchondrosis merits the title of universal joint more than any other joint in the body, for it permits all the movements of an enarthrosis (a ball-and-socket joint) with, in addition, the capacity for movement in its vertical axis in response to tension and compression. The focal point, so to speak, around which these movements occur, is the nucleus pulposus embedded in, and maintained in a state of compression by, the cartilaginous annulus fibrosus. The nucleus, which is a semi-fluid structure, has expansile properties when released from its surrounding compression. Mechanically it is said to behave like an incompressible fluid which, with the alterations in shape of the surrounding annulus, helps to distribute pressure more evenly from one vertebra to another. The annulus certainly undergoes significant changes of shape in different movements on the spine, and I think it is useful to think of the nucleus as a resilient ball around which such movements occur.

Each individual intervertebral disk is firmly attached to the vertebra above and the vertebra below through the cartilaginous plate which covers the vertebral surface, the peripheral portion of which is ossified to form the vertebral epiphyseal ring. The annulus is composed of fibro-cartilage. The thin epiphyseal plate is, of course, hyaline cartilage. In the adult it forms a very thin lining covering the exposed cancellous bone of the vertebral surface within the epiphyseal ring, and is the only protection which the cancellous bone has from the variable pressure changes produced within the intervertebral diska point of some pathological importance. Other attachments of the disks are provided by the anterior and posterior common ligaments, of which the former are the denser. Under pressure each disk bulges somewhat at its periphery (but it is of interest that uniform pressure of several times $g$ does not cause any appreciable diminution of thickness). With changes of movement these bulges vary in amount. The continuous and repeated pressure or tension which they cause upon the peripheral fibrous tissue provokes the characteristic changes of senility; that is, the beak-like ossification from the vertebral bodies or osteo-arthritic spurs seen so readily at the front and side of the disks in skiagrams.
The resilience of the intervertebral disks and consequently their flexibility depend, it is said, upon their water content, which diminishes with age. Thus these bulges tend to persist, and yet for the same reason to become smaller. Ossification or calcification around the bulging disks is less likely to occur posteriorly, but it may occur. One should note by comparison the lack of bulging seen in the spines of young patients with ankylosing spondylitis, in which ossification follows the straight line of the peripheral ligaments. As Schmorl has shown, disk bulging may occur also in a vertical direction when there has been a break through the cartilaginous plate referred to previously. In such cases herniation of disk substance occurs into the cancellous vertebral body. A more generalized vertical bulging of intervertebral disk may occur in certain metabolic diseases such as hyperparathyroidism or senile decalcifying spondylitis in which the vertebral bony substance is abnormally soft. The intervertebral disks are relatively avascular structures; they degenerate and become thinner with loss of their characteristic properties when the nutrition of their surrounding tissues is interfered with. This is seen characteristically in tuberculosis, in which the disease starts in the neighbouring bone and produces early degeneration of the disk before it is invaded by tuberculous granulation tissue. Similar disk degeneration may occur as the result of trauma. Absence of the characteristic changes in the vertebral bodies nearby distinguishes the two conditions. Cartilaginous tumours arising from remnants of the notochord are described, but it is probable that many of those described in reports published before 1932 were, in fact, protrusions of disk substance. Although nerve fibres from the spinal nerves are seen to pass on the posterior surfaces of the vertebral bodies, it is probable that the disks are relatively insensitive, and that symptoms produced by pathological changes are due to irritation of surrounding tissues. Particularly is this true posteriorly and laterally (in cases of posterior protrusion) because of the presence of the meningeal structures, the spinal nerve roots, and the spinal nerves. The relation of the latter, of course, accounts for the segmental distribution of symptoms and signs.

We have to consider first the effects produced by the local irritative change, and secondly a profound derangement of all the anatomical structures which go to maintain the stability of the spine, particularly in the region in which the lesion occurs. While, therefore, there may be nerve-root oedema and fibrosis, derangements of the posterior apophyseal elements of the intervertebral joint 
complex, tearing of ligamentous attachments, and so on, nevertheless we must recognize the great reflex disturbances in the muscular system which any joint derangement will cause.

\section{Clinical Features}

As has been stated previously, lesions of the disks of one sort or another are common. They are often present without giving rise to any symptoms whatsoever. This applies equally to posterior protrusion, which Schmorl has shown to be present in 35 per cent. of all necropsy material studied by him. While these protrusions may not cause any symptoms, those which do have no absolutely clear clinical syndrome. Identical symptoms and signs can be found in other pathological processes, remote from the intervertebral disk, as well as in diseases of the disks without posterior protrusion.

Radiologically there is no characteristic picture. Posterior disk protrusion may be found with a normal skiagram; a skiagram showing disk degeneration does not necessarily indicate that there is mechanical disk pressure upon the neighbouring nerve roots.

In considering the clinical problem, it is useful to apply the simile of an explosive incident. There is a train of events or conditions leading to the setting of the fuse, or the pulling of the trigger, and to the resultant catastrophe. The background of our problem is often a background of physical and psychological defect, incompetent spinal musculature with poor postural control, and an inefficient use of body mechanics in performing the normal tasks. Stressing the lumbar spine by indirect violence while it is in flexion is doubtless one causal factor; there are probably many other inherent defects of the individual which permit the trigger release, namely, the extrusion of disk substance into the spinal canal or into the intervertebral foramen. As a clinical problem, it is difficult to sort out those explosive symptoms and signs which are due purely to the trigger release, and those which are secondary. The kinking or traction of a nerve root and the meningeal coverings causes pain of segmental distribution, paraesthesia, and disturbances of temperature sense. The motor effect will be spasm. The irritative signs are succeeded by those which are paralytic, as neurone damage becomes more complete; analgesia and anaesthesia of segmental distribution are associated with muscular atrophy and contracture, loss of peripheral reflex, and even loçalized paralysis. The irritative signs are increased by movements of the spine which aggravate the mechanical involvement of the nerve root (such as the flexion of the spine, which automatically occurs when the hip is flexed with the straight knee, or when meningeal tension is accentuated by flexion of the neck), and by all the circumstances which may cause a rise of cerebrospinal fluid pressure, such as coughing or sneezing, straining at stool, or jugular compression. It may be said that much of the explosive effect of such disk lesions is due to the causes $I$ have just enumerated, but in addition there is an overflow of impulses from the affected segment into neighbouring segments, while muscle spasm itself is painful and provokes its own very widespread reflex effects, and, of these, scoliosis is a visible sign. Scoliosis is of a special type, and is best described as a list which transfers body-weight to the opposite limb; less often the list is to the same side (homolateral) and occasionally it alternates almost at will from one side to the other. As I have previously shown elsewhere (Capener, 1933), the one curve is not the mirror image of the other, for in alternating sciatic scoliosis the contralateral curve takes place low down in the spine, whereas the homolateral curve appears to take place at a higher level.

The segmental distribution of pain is not itself sufficient to justify the diagnosis of disk retropulsion, for it is known that stimulation in any part of a spinal nerve segment may produce pain referred throughout the rest of the segment. In experimental work Lewis and Kellgren (1939) have suggested that irritative injections into the supraspinous ligaments may reflexly cause sciatic pain, but recent work of Sinclair and his colleagues (1948) suggests that these effects are the result of direct stimulation of the posterior primary divisions of spinal nerves. The same can be said about many of the other segmental signs. Nevertheless, the persistence of purely segmental signs and symptoms should lead to the serious consideration of the diagnosis of intervertebral disk retropulsion, and this point should, I maintain, add particular emphasis to the form of treatment which I advocate and which as I will show, deals primarily with the widespread effects of such disk disturbances and of other causes of -sciatica.

\section{Treatment}

In derangements of the knee our programme is one of early support, graduated movements, and re-training. Such a programme is equally necessary in the spine.

The primary treatment should be the application of physiological rest. This does not mean allowing the patient to lie in bed in any position he likes. It does mean control in a physiological position with graduated activity as the pathological process 
and clinical signs subside. When the symptoms are of the greatest severity, the spine and lower extremity must be controlled with practically complete immobilization in recumbency. The methods vary from one clinic to another-the use of a plaster jacket with traction upon the affected lower extremity, the use of a plaster spica or even a plaster bed, including the spine and both lower extremities, may be chosen. For most cases, however, the plaster jacket is the most satisfactory. This gives only relative immobilization of the lumbar spine, and does not extend above the nipple line but does come well down on to the pelvis at the back and front. It is applied with slight steadying head traction. It does not support the spine, but it does provide an important aid to the muscular system of the lower spine. After the jacket is applied, rest in recumbency with a knee pillow is ordered for a few days; but is steadily diminished as symptoms subside. Radiant heat and massage for the lower extremity will assist in relieving pain and muscle spasm. Then a graduated course of remedial exercises is started in order to re-train the whole extensor mechanism, including the gluteal muscles, action of which stimulates the spinal muscles synergically. When the acute symptoms have subsided, the patient will still be made to take a considerable mid-day rest in recumbency so as to relieve the readily fatigable spinal muscles. The plaster jacket is retained for from five to twelve weeks according to the severity of the symptoms and the speed with which they subside. In patients with sciatic scoliosis the jacket may require changing, for until spasm is relieved one does not attempt to correct the early deformity. After the necessary period, the jacket is split in the front midline, and can be removed increasingly for the further elaboration of spinal movements. Usually at this time it may be slipped off at night and for baths. Remedial exercises at this stage should include stretching the hamstring muscles. This is not done by forward flexion of the trunk on the hips. The patient carries out straight leg raising in the supine position with a passive support of gradually increasing height, stopping short of pain. For many months afterwards he should be warned not to bend forwards with the knees extended, nor should he sit in bed in a similar position. He must avoid lifting heavy weights from the ground and instead should get " under his weights" by bending the knees.

The course of treatment will extend over a period of three months, and the clinician should by then have been able to decide in most cases whether or not the patient is going to be relieved of his disability without further intervention. The more wide- spread secondary effects will have subsided, and even the purely segmental signs may have disappeared. The important thing is that in those few cases which do not respond, the neurological signs will have become relatively isolated and clearly defined, and a decision can then be more carefully made as to those cases which can still be treated conservatively, and those which need operation. Always, however, must be borne in mind the fact that occasionally the signs of neurological damage are so complete and paralytic from the outset that operation is almost an emergency.

\section{Results of Treatment}

Conservative Treatment.-My associate, Mr. F. C. Durbin (1948), has published the results of an investigation he has carried out into the use of the plaster-jacket treatment for sciatica, in my orthopaedic service at the Princess Elizabeth Orthopaedic Hospital, Exeter, during the years 1936-1945 inclusive. During this ten-year period, 525 patients with sciatic pain were treated. In the investigation there were selected 225 patients who, because of segmental neurological change (such as absent ankle jerk, sensory loss, or muscle wasting) might justifiably be considered to have suffered from intervertebral disk retropulsion. Follow-up was possible in 147 patients of whom there were 82 men and 65 women. Of the cases, 65 per cent. were between the ages of 20 and 40 . The plaster-jacket method of treatment was used in 123 patients, and of these 43 were cured, 35 were relieved, and 45 were not relieved, 16 of the last group being subsequently treated by laminectomy. By "cured" is meant complete relief of pain with no recurrence over a period of from two to ten years, the patients continuing in their normal occupations. By "relieved" is meant persistence of very little residual pain, the patient doing heavy duties though often sparing himself the lifting of heavy weights. By " not relieved" is meant that the pain was never completely controlled, or that there were many recurrences requiring rest in bed for more than a few days. Two interesting points showed up: first, that the duration of symptoms before treatment influenced the likelihopd of relief. In the failed group, when symptoms had been present for more than a year the figure varied between 41 and 51 per cent. The other point is that loss of ankle jerk, and hypo-aesthesia both tend to persist. On the other hand, simple diminution of an ankle jerk often disappears when symptoms are relieved.

I do not think Mr: Durbin would object if I said that he approached the problem with scepticism in 1946. Allowing for this and the fact that this work 
and the after-treatment was, for six of those years, carried out under the very difficult conditions of wartime, the results are of considerable interest. Few figures have been published dealing with the results of the conservative treatment of sciatica; at the same time these figures cannot be considered as final, for there are many variable factors. An illustration of what is being done at Exeter along these lines is given in the Table.

\section{TABLE}

PLASTER JACKETS APPLIED IN THE TREATMENT OF SCIATIC PAIN*

(At the Princess Elizabeth Orthopaedic Hospital, Exeter)

\begin{tabular}{r|r}
\hline 1942 & 54 \\
1943 & 68 \\
1944 & 132 \\
1945 & 154 \\
1946 & 162 \\
1947 & 178
\end{tabular}

* During the first six months of 1948 the number of treatments by plaster jacket is still rising, for 132 have been applied.

Operative Treatment.-An enormous amount of experience has been gained by both neurosurgeons and orthopaedic surgeons in the United States of America. It is interesting to note the changing point of view. That simple removal of protruded intervertebral disk substance is by itself not giving entirely satisfactory results is indicated by the increasing number of combined operations, including lumbo-sacral fusion, which are being reported. A disturbing report is contained in the paper by Aitken and Bradford (1947), in which the experience of one insurance company is reported and 170 cases operated upon between 1940 and 1944 are reviewed. These were all compensation cases, and that this fact influenced the end-result is not denied. Nevertheless, as the authors state, the period in question was one of intense industrial activity because of the war, with every encouragement for patients to work even with a partial disability. The operations were not carried out in a single institution, but were the work of many hospitals and surgeons. Only 13 per cent. were symptom-free and capable of performing heavy laborious work after the operation. Only 17 per cent. were in the group that we would call relieved; 25 per cent. had pain and were only able to do light work; 42 per cent. were classed as bad results; and there was a 3 per cent. mortality rate. Placing the first and second groups together as good results, the comparison of 30 per cent. good and 42 per cent. failures is indeed striking. Two additional points in this analysis are of interest, namely, that the percentage of error in diagnosis, with present diagnostic criteria, was about 40 per cent., and that secondary operations were required in 24 per cent. of all cases.

\section{Conclusions}

There are many lesions of the intervertebral disk besides posterior protrusion. There are no unmistakable signs, clinically or radiologically, of disk protrusion. The most. suggestive signs of this condition are also given by other pathological lesions of the vertebral column and spinal cord. In the analysis of the clinical state and in planning treatment, one must consider three elements: first, the patient as a whole; secondly, the immediate local lesion; and thirdly, the widespread physiological disintegration. While we all probably recognize in various degrees the need to treat the patient, there is a common failure to treat adequatefy the explosive effects produced by such spinal derangements. In advocating the use of the plaster jacket for applying rest, one should emphasize the importance of such treatment early, not only because it is the best method of treating all three elements of the problem, but also because it enables us to separate that group of individuals who have a more severe organic lesion likely to require operative intervention, which in any case should be less often needed if this treatment and its after-care are diligently pursued.

I have thought it of value to quote some figures from an investigation of a considerable series in which conservative treatment has been given. At the Princess Elizabeth Orthopaedic Hospital, we have had a relatively large experience. As Geoffrey Jefferson has said to me: " Experience may merely be the repetition of the same error." I hope this is not so in the present example. At any rate it would be relatively harmless.

\section{REFERENCES}

Aitken, A. P., and Bradford, C. H. (1947). Amer. J. Surg., 73, 365.

Capener, N. (1933). Proc. R. Soc. Med., 26, 425

(1944). Annals of the Rheumatic Diseases, 4, 29.

Durbin, F. C. (1948). J. Bone Jt. Surg., 303, 487.

Lewis, T., and Kellgren, J. H. (1939). Clin. Sci., 4, 47.

Sinclair, D. C., Feindel, W. H., Weddell, G., and Falconer, M. A. (1948). J. Bone Jt. Surg., 303, 515 .

Signification Clinique et Traitement des Lésions du Disque Intervertébral

RÉSUMÉ ET CONCLUSIONS

Cette article concernant le "Problème du Disque" constitue un complément à l'étude anatomique et mécanique de la région lombo-sacrée, publiée dans les Ann. Rheum. Dis., 1944, 4, 29. La théorie est que, quelle que soit l'importance du rôle de la rétropulsion d'un disque intervertébral dans la sciatique, elle est néanmoins accompagnée par un nombre important de manifestations qui doivent être soumises à un traitement prolongé avant que l'on soit en droit de procéder à une intervention chirurgicale. 